\title{
A New Satellite Image Enhancement Method using Wavelet Transformation and Morphological Filtering
}

\author{
Gauri Rode ${ }^{1}$, V. K. Shandilya ${ }^{2}$, Dr. S. A. Ladhake ${ }^{3}$ \\ ${ }^{1}$ M. E. $2^{\text {nd }}$ year, Computer Science and Engineering, Sipna College of Engineering and Technology, Amravati, Maharashtra, India \\ ${ }^{2}$ Associate Professor in Computer Science and Engineering, Sipna College of Engineering and Technology, Amravati, Maharashtra, India \\ ${ }^{3}$ Principal, Sipna College of Engineering and Technology, Amravati, Maharashtra, India
}

\begin{abstract}
Many enhancement methods have been proposed earlier to enhance satellite images. Most of them have critical and more advance steps still those methods unable to give satisfied result. One of the necessary issues regarding to the satellite images is to sharpen the edges, as edges are helpful in identifying important information from the images. So new method is proposed in this paper regarding to detect and enhance the edges of satellite images. Also the noise removal and preservation of useful information are other important aspects of this work. In this work Gabor filter is used for efficiently detects the edges from image, while morphological filtering applies on that detected edges to sharpen it. This method gives better qualitative and quantitative result compare to the well known state-of- the-art techniques.
\end{abstract}

Keywords: Image enhancement, Gabor filter, wavelet transform, morphological filtering, remote sensing.

\section{Introduction}

Image processing in which we are working on the image or video to make it more informative. It is a quickly growing technology in today's era; its applications are used in various trades. As we know it is applicable in wide area like Remote Sensing, Medical Application etc. In image processing the most appealing area is image enhancement. The motive behind image enhancement is to bring out detail that is not visible clearly or simply to highlight important features of an image. Enhancement of the image is necessary to improve the visibility of the image which indirectly means that to remove unwanted noise, to improve contrast, enhance resolution and to find more details. Ultimate aim of this method is to bring additional detail from image. [1, 2]

The meaning of enhancement is the alteration of the appearance of an image in such a way that the information contained in that image is more readily interpreted visually. This method used to improve a satellite image which has complete information but is not fully visible. Enhancement method is used on satellite images to make it more informative. These images have been use mostly for land cover identification and classification various land surface taken from satellite. The data which taken from remotely sensed source is used in many applications like environment, forestry, agriculture, geology, hydrology and many others. Therefore such remotely sensed images used in various fields, it has different roles to perform. Image enhancement is used for making an image more interpretable for all types of applications. [3]

One of the necessary issues regarding to the satellite images is to sharpen the edges, as edges are helpful in identifying important information from the images. So new method is proposed in this work regarding to detect and enhance the edges of satellite images. Many important image processing applications such as object recognition, segmentation and robot vision depend on the how accurately the edges are detected from images. Therefore edges contain important information in image. [4]

After processing the satellite images, it further used for crop production forecast, , disaster management, forest cover and type mapping, weather forecasting, watershed development and monitoring and many other. [2]

There are many traditional filters are available and by using them edge enhancement filtering is carried out. These kinds of filters contain some problem when it applies on noisy images. Therefore it is necessary to find other alternative method which can deal with such problems also. A variety of methods have been previously proposed to solve the edge preserving problem.

Noise removal and preservation of useful information from an image are also important aspects of image enhancement. This work is mainly focused on the extraction of the important information from the image and number of operation done for enhancing the image. [5]

Image enhancement method is classified into spatial domain method and frequency domain method. Spatial based enhancement operations are performed in order to increase or decrease the image brightness, contrast or the distribution of the grey levels. Due to this the pixel value of the output image will be change according to the transformation methods applied on the input values.

Whereas the spatial domain methods contains bilateral filter, which have drawbacks of blur or produce distortion in image. Wavelet based edge detection method has better visual 


\section{International Journal of Science and Research (IJSR) \\ ISSN (Online): 2319-7064 \\ Index Copernicus Value (2013): 6.14 | Impact Factor (2015): 6.391}

quality than the other existing edge detectors such as canny, sobel and other. [6]

Wavelets can be used to extract the information from a images. Wavelet transforms are different types such as continuous wavelet, discrete wavelet and multi resolution based transforms. Sharpness of image is done by using wavelet coefficient; also the satellite image resolution enhancement has been done in wavelet domain. [7]

One of the most important wavelet transform method is discrete wavelet transform method. In this wavelet transform method divides the images in to different frequency subbands i.e. Low frequency band (LL), high frequency band (HH) and Mid Frequency Band (LH, HL). There are also some techniques for enhancing a satellite image such as histogram equalization technique, discrete cosine transform technique etc [3]

\section{Proposed Method}

The main objective of this work is to enhance satellite images. A new image enhancement technique is instigated for making satellite images more informative and helping to achieve the goal of image interpretation. Edge in the image helps to identify useful information, due to this reason edge enhancement focus mainly.

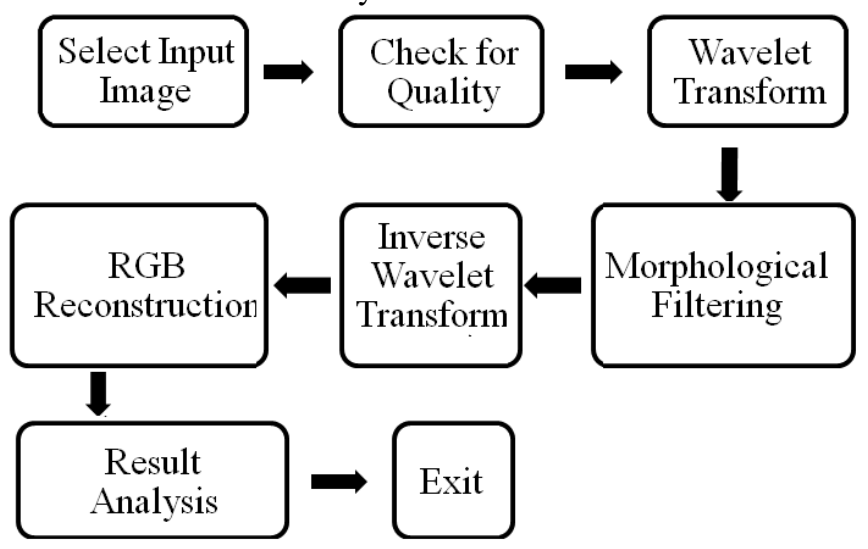

Figure 1 : Block Diagram of Proposed Method

\subsection{Select Input Image}

Initial step is to select satellite image as an input. After that the input low contrast color image is decomposed into R, G, B color component. Separately shows these R, G, B value by using histogram.

\subsection{Check for Quality}

The input satellite images need to enhance or not, we can find out it in this step. For which we have to first calculate the mean intensity of the image. Mean Intensity of the image is the average of the intensities of all pixels in an image. We calculate this in matlab by using STAT function.

First find the value of, $n=$ length $(x)$

Next calculate the value of, mean $=\operatorname{sum}(x) / n$

If the mean intensity is greater than 0.5 then we consider as quality image which does not need to enhance but if it is less than or equal to 0.5 then it is low vision image need to enhance.

\subsection{Wavelet Transform}

Gabor filters are directly related to Gabor wavelets, since they can be designed for number of dilations and rotations. In general the Gabor wavelets are not uses directly because of its biorthogonal property, it require more time for computation. Therefore one filter bank created which consist of Gabor filters with various scales and rotations.

It is the form of sine wave modulated by the Gaussian coefficient. In image processing a Gabor filter is a linear filter mainly used for edge detection purpose, also there are used in various other applications. As the frequency and orientation representations of Gabor filters are similar to those of the human visual system so it is appropriate for texture representation and discrimination in image processing. In our work Gabor filter play key role it is used for edge detection and enhancement purpose.

A Gabor filter is a linear filter whose impulse response is defined by a harmonic function multiplied by a Gaussian function

$$
\begin{aligned}
& x_{\text {smooth }}(m, n)=x(m, n) \otimes h(m, n) \\
& x_{\text {edge }}(m, n)=x(m, n)-x_{\text {smooth }}(m, n)
\end{aligned}
$$

After calculating the value of $\mathrm{x}_{\text {smooth }}$ in above equation, in next step subtract $x_{\text {smooth }}$ from $x$ gives the image edge $x_{\text {edge }}$, in this way it detect the edges from the image. After that the detected edges need to sharpen more, therefore the equation used for this.

The detected edges from the image can be used for edge enhancement if it is added back to the original image signal as shown in Eqn.

$$
x \operatorname{sharp}(m, n)=x(m, n)+k[\text { xedge }(m, n)]
$$

Where $\mathrm{k}$ is a scaling constant we called it as an enhancement factor.

Value of $\mathrm{k}$ is user defines one, but in our work we have set value for it. As we have already found the value of mean intensity in second step, by using that we select range for $\mathrm{k}$.

Table 1: New K value range

\begin{tabular}{|c|c|c|}
\hline Sr. No. & Mean Intensity & $K$ \\
\hline 1 & 0 to $<=0.20$ & 0.7 \\
\hline 2 & 0.20 to $<0.40$ & 0.8 \\
\hline 3 & 0.40 to $<0.60$ & 0.85 \\
\hline 4 & 0.60 to $<=1$ & 0.9 \\
\hline
\end{tabular}

If mean intensity is in the range 0 to 0.20 then value of $\mathrm{k}$ is set as 0.7 , in between greater than 0.20 to 0.40 then the $\mathrm{k}$ is set to 0.8 , greater than 0.40 to 0.60 then $\mathrm{k}$ is set to 0.85 , greater than 0.60 to 1 then value of $\mathrm{k}$ is 0.9 . This is our new work regarding to scaling constant $\mathrm{k}$. In the above table 1 the value of $\mathrm{k}$ is illustrated for better understanding. 
Gabor wavelet transform apply on satellite images for find fine information and then used in many applications such as geosciences studies, astronomy, forestry and geographical information systems.

\subsection{Morphological Filtering}

After detecting the positions of the edges and then applying a morphological filtering on it. A morphological filter is a non linear filter used to sharpen these detected edges. It is used for solving the numerous image processing problems, therefore it is important aspect. One of the most important aspects of morphological image processing is for solving the image sharpening problem.

\subsection{Inverse Wavelet Transform}

After that this enhanced frequency components have to be reconstructed using inverse wavelet transform.

\subsection{RGB Reconstruction}

The R, G, B value reconstructed back in this step. Also for finding the accurate enhance image we created one loop here where different value we get. To execute loop initially we can select one random value $\mathrm{R}$, then the pixel value greater than that value is consider as object pixel while pixel value less than that value consider as background pixels. Then find the average of object pixels i.e. G1 and average of background pixels i.e. G2. By calculating average of G1 and G2, we get new value of $R$. Now this new value of R use in initial step, continue execution until we get accurate result. After execution of this steps we get enhance satellite image.

\section{Results and Discussion}

In this paper we have discussed about new method of satellite image enhancement. Demonstrate proposed algorithm on several satellite images to prove its efficiency than other existing methods. Peak signal to noise ratio is calculate by following formula;

$$
P S N R=10 \log _{10}\left(\frac{R^{2}}{M S E}\right)
$$

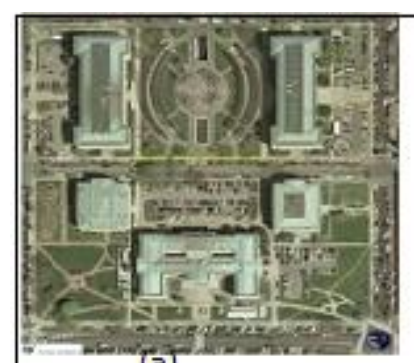

(a)

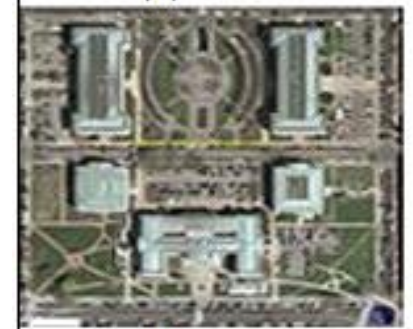

(c)

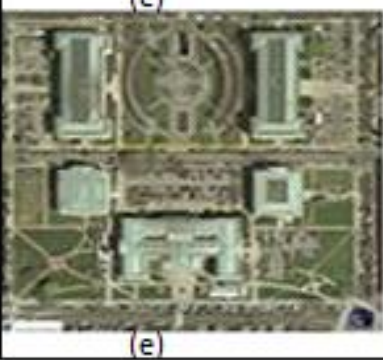

(e)

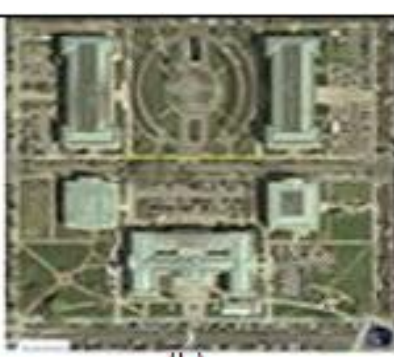

(b)

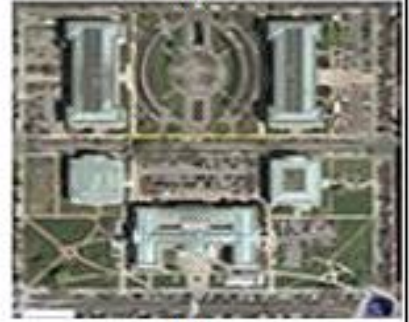

(d)

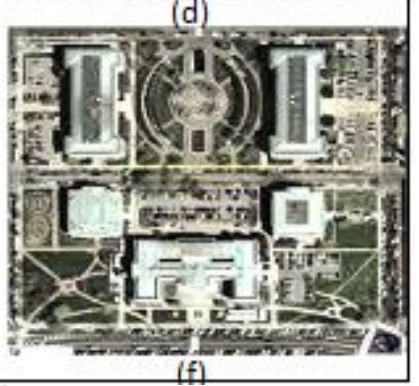

(f)

Figure 2: a. Original Washington DC image, b. Input image, c. SWT-RE, d. DWT-RE, e. DT-CWT-RE, f. Proposed method

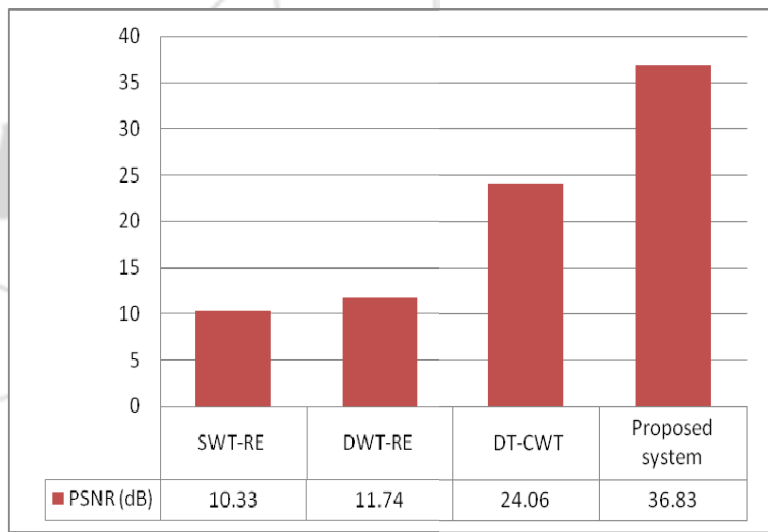

Figure 3: PSNR (Decibels) results of proposed technique compared with conventional and some state-of-art techniques.

Table 3: Computational Time result for LANDSAT 8 image

Table 2: PSNR value comparison for satellite image

\begin{tabular}{|c|c|}
\hline Method & PSNR $(\mathrm{dB})$ \\
\hline SWT-RE & 10.33 \\
\hline DWT-RE & 11.74 \\
\hline DT-CWT & 24.06 \\
\hline Proposed system & 36.83 \\
\hline
\end{tabular}

In the above table 2 show comparative value of PSNR by using different method on Washington DC image. As proposed method value is greater which indicates that the image quality is good. In figure 2 shows that different methods apply on Washington DC image. Figure 3 illustrated the PSNR results comparison with existing state-of-art methods. [8]

\begin{tabular}{|c|c|}
\hline Method & Computational Time (sec) \\
\hline Low pass filter & 2.68 \\
\hline Gan [9] & 2.89 \\
\hline Coulange [10] & 2.55 \\
\hline Discrete wavelet transform [11] & 2.5185 \\
\hline Proposed system & 1.85 \\
\hline
\end{tabular}




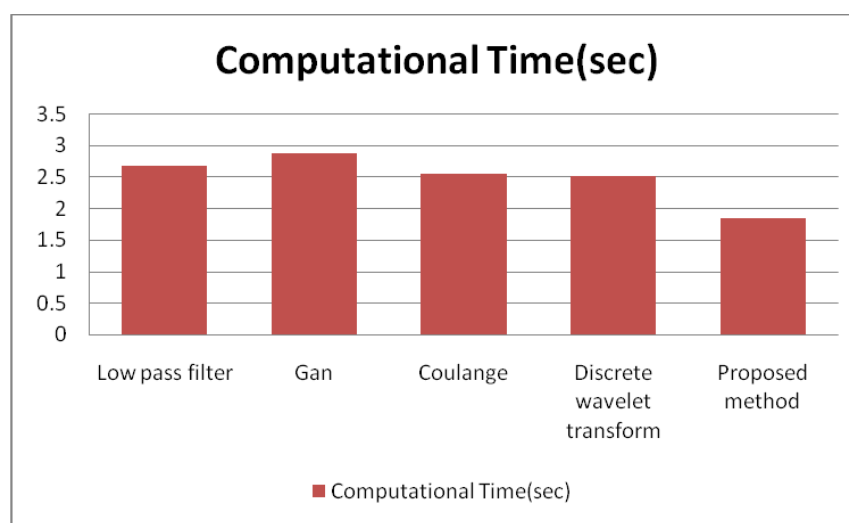

Figure 4: Computational Time(sec) comparison with other existing methods

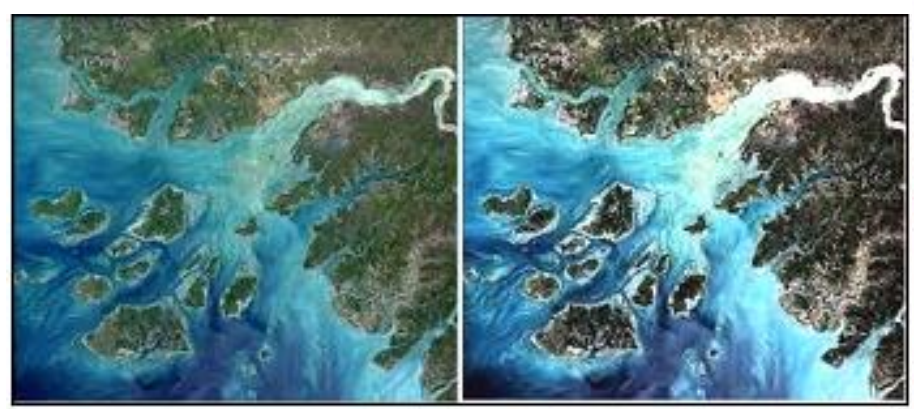

Figure 5: Image from LANDSAT 8 Satellite

Computational time require for image taken from LANDSAT 8 satellite is calculated, in figure 5 shows original and enhance satellite image. Results mention in table 3, less computational time require means proposed method is faster in execution with other methods, therefore save time. [11] In figure 4 we can see clear difference as minimum time require for proposed method as compare to other methods.

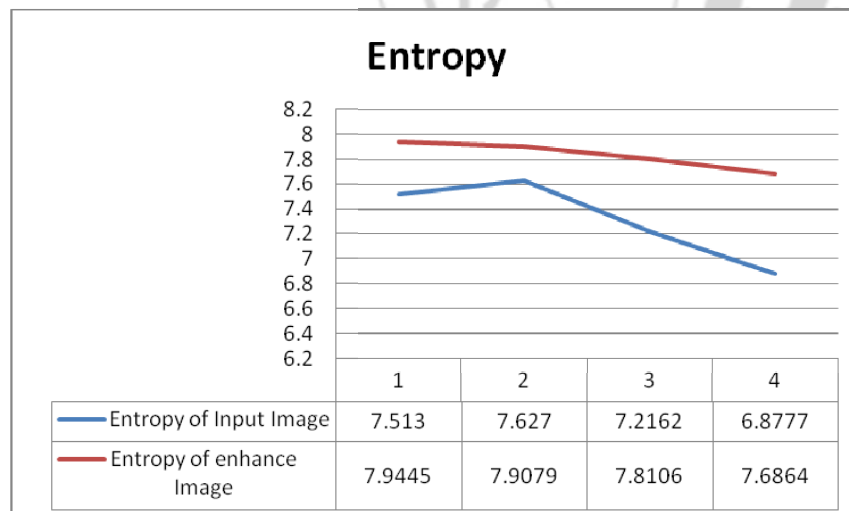

Figure 6: Entropy value comparison with input image and enhance image of figure 7

Entropy value gives idea about the amount of information coded in an image. Low entropy of enhance image than input image shows that it loss some information or if entropy is increases in enhance image, so is its information content. We have taken different input image as shown in figure 7 and after enhancing input image by using proposed method shows in right side. In figure 6 shows comparison of entropy of input image with entropy of enhance image, from that we get clear idea that entropy of enhance image is increased by using our proposed method.

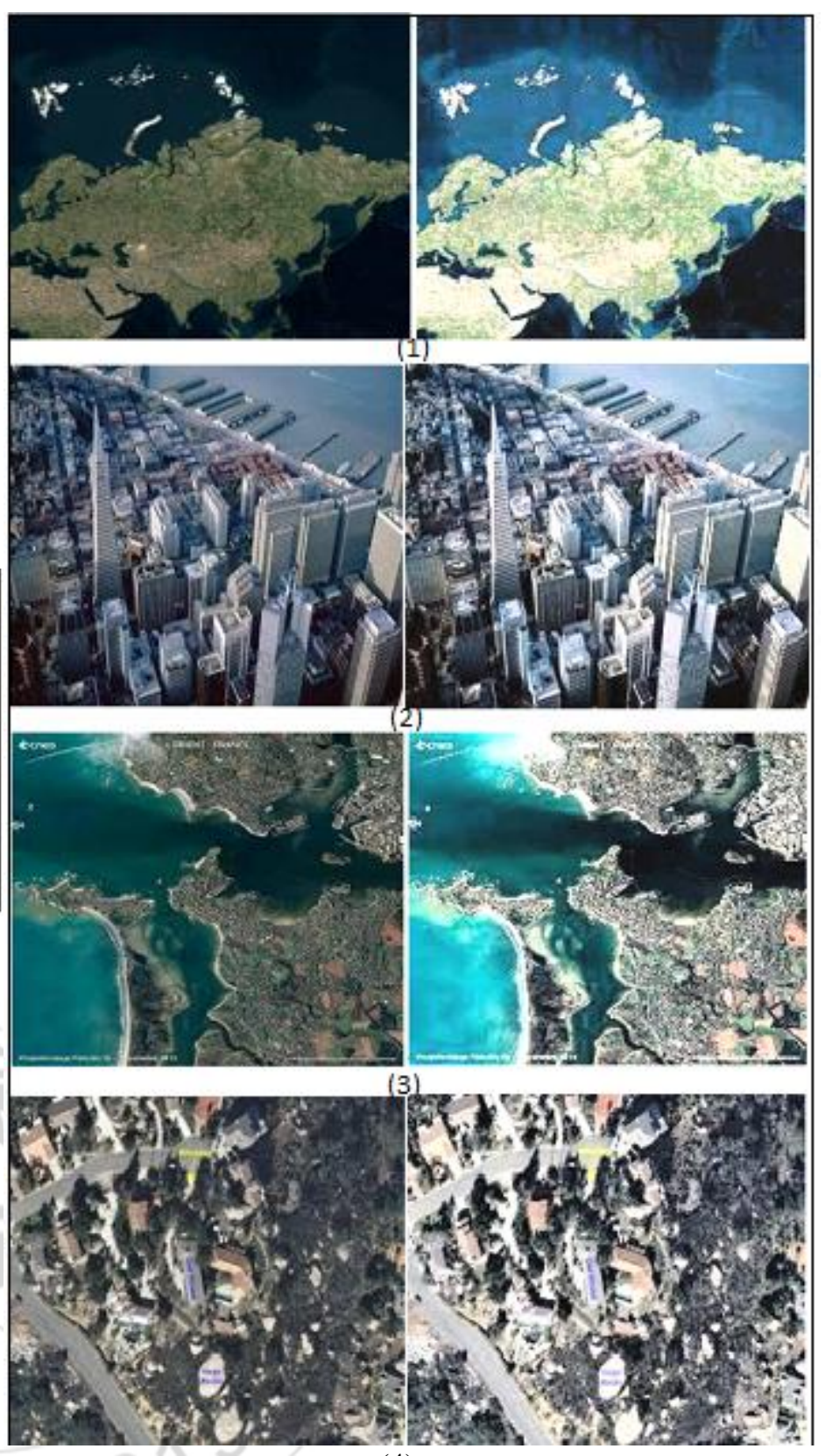

(4)

Figure 7: Left side images are input image and right side images are enhance images

Satellite image enhancement using our proposed method shows better quantitative and qualitative results, we can see in figure 7.

\section{Conclusion}

This paper gives the information about the enhancement of satellite images. We have implemented the method to improve the quality of satellite images so that it becomes more informative. The gabor filter is very effective in detecting the edges from low contrast satellite images and detected edges is sharpen by using morphological filtering process. The visual examples shown in above figures, have demonstrated that the proposed method was significantly better than many other well-known methods in respect of edge and fine detail restoration. This work will helpful to study the effective method for satellite image enhancement. 


\section{International Journal of Science and Research (IJSR) \\ ISSN (Online): 2319-7064}

Index Copernicus Value (2013): 6.14 | Impact Factor (2015): 6.391

The proposed system verify by both quantitative and qualitative parameter. It takes less computational time as compare to other methods. In the result analysis stage it shows that the PSNR and entropy value of image which is better than existing methods. In future different wavelet transform method can be used for the satellite image enhancement.

\section{References}

[1] P. Suganya, N. Mohanapriya, B. Kalaavathi, "Satellite image resolution enhancement using multi wavelet transform and comparison of interpolation techniques", International Journal of Research in Engineering and Technology, eISSN: 2319-1163 | pISSN: 2321-7308, Volume: 03 Special Issue: 07 | May-2014.

[2] Vaibhav R. Pandit, R. J. Bhiwani, "Image Fusion in Remote Sensing Applications: A Review", International Journal of Computer Applications (0975 - 8887) Volume 120 - No.10, June 2015.

[3] Arya P Unni, "Satellite Image Enhancement Using 2D Level DWT", International Journal of Engineering Research \& Technology (IJERT), ISSN: 2278-0181, Vol. 3 Issue 3, March 2014.

[4] Abdul Rasak Zubair, Olasebikan Alade Fakolujo, "Image Edge Detection and Image Edge Enhancement: Numerical Experiment on High Pass Spatial Filtering”, International Journal of Computer and Information Technology (ISSN: 2279 - 0764) Volume 03 - Issue 04, July 2014.

[5] Dr. Muna F. Al-Samaraie and Dr. Nedhal Abdul Majied Al Saiyd , "Colored Satellites Image Enhancement Using Wavelet and Threshold Decomposition", IJCSI International Journal of Computer Science Issues, Vol. 8, Issue 5, No 3, September 2011.

[6] DEVI.S, JINI CHERIYAN, "Image Enhancement Using Guided Image Filter and Wavelet Based Edge Detection", International Journal of Modern Engineering Research (IJMER), Vol. 3, Issue.3, May.-June. 2012 pp-1702-1706.

[7] Aparna Gupta, Miss Sonika, "Resolution Enhancement of Satellite Images with Interpolation using DWT-SWT wavelet domain", International Research Journal of Engineering and Technology (IRJET) e-ISSN: 2395 0056, p-ISSN: 2395-0072, Volume: 02 Issue: 07 | Oct2015.

[8] P.Subbulakshmi, S.Bhavani, "Enhancement of Satellite Images Resolution Using Dual-Tree Complex Wavelet Transform", International journal of innovative research in electrical, electronics, instrumentation and control engineering, vol. 1, issue 9, December 2013.

[9] J. Gan and D. Taubman, "A content-adaptive waveletlike transform for aliasing suppression in image and video compression", IEEE International Conference on Image Processing, pp. 3821-3824, Nov.2009.

[10]B.Coulange and L.Moisan, "An aliasing detection algorithm based on suspicious colocalizations of Fourier coefficients", In Proc.IEEE Int Conf.on image processing, Hong Kong, China, pp.2013-16, sep-2010.

[11] Mote Ashwini G. Pachkor Kavita V. Patil Samruddhi S., "Satellite Image Analysis Using DWT and Structural
Texture Similarity Metrics", International Journal of Computer Application, Issue 4, Volume 5 (Sep - Oct 2014). 